\title{
The COVID-19 PANDEMIC: A Rapid Global response for Children with Cancer from SIOP, COG, SIOP-E, SIOP-PODC, IPSO, PROS, CCI and St Jude Global
}

\author{
Michael Sullivan ${ }^{1}$, Eric Bouffet ${ }^{1}$, Carlos Rodriguez-Galindo ${ }^{1}$, Sandra Luna-Fineman ${ }^{1}$, \\ Muhammad Saghir Khan ${ }^{1}$, Pam Kearns ${ }^{1}$, Douglas S. Hawkins ${ }^{1}$, Julia Challinor ${ }^{1}$, Lisa \\ Morrissey $^{1}$, Jörg Fuchs ${ }^{1}$, Karen Marcus ${ }^{1}$, Adriana Balduzzi ${ }^{1}$, Luisa Basset-Salom ${ }^{1}$, Miguela \\ Caniza $^{1}$, Justin N. Baker ${ }^{1}$, Rejin Kebudi ${ }^{1}$, Laila Hessissen ${ }^{1}$, Richard Sullivan ${ }^{1}$, and Kathy \\ Pritchard-Jones ${ }^{2}$ \\ ${ }^{1}$ Affiliation not available \\ ${ }^{2}$ University College London
}

April 29, 2020

\begin{abstract}
The COVID-19 pandemic is one of the most serious global challenges to delivering affordable and equitable treatment to children with cancer we have witnessed in the last few decades. This Special Report aims to summarise general principles for continuing multi-disciplinary care during the SARS-CoV-2 (COVID-19) pandemic. With contributions from the leadership of the International Society for Paediatric Oncology (SIOP), Children's Oncology Group (COG), St Jude Global programme and Childhood Cancer International, we have sought to provide a framework for healthcare teams caring for children with cancer during the pandemic. We anticipate the burden will fall particularly heavily on children, their families and cancer services in low- and middle- income countries. Therefore, we have brought together the relevant clinical leads from SIOP- Europe, COG and SIOP-PODC (Pediatric Oncology in Developing Countries) to focus on the six most curable cancers that are part of the WHO Global Initiative in Childhood Cancer. We provide some practical advice for adapting diagnostic and treatment protocols for children with cancer during the pandemic, the measures taken to contain it (e.g. extreme social distancing) and how to prepare for the anticipated recovery period.
\end{abstract}

Corresponding author: Professor Kathy Pritchard-Jones, Professor of Paediatric Oncology. President, International Society of Paediatric Oncology (SIOP), UCL Great Ormond Street Institute of Child Health. University College London 30 Guilford Street, London, WC1N 1EH UK Email: k. pritchard-jones@ucl.ac.uk

\section{Hosted file}

Sullivan et al COVID Pandemic a rapid global response preprint FINAL 27-04-2020.pdf available at https://authorea.com/users/5588/articles/445428-the-covid-19-pandemic-a-rapid-global-responsefor-children-with-cancer-from-siop-cog-siop-e-siop-podc-ipso-pros-cci-and-st-jude-global 